\title{
Ontology-based User Modeling for Knowledge Management Systems
}

\author{
Liana Razmerita, Albert Angehrn ${ }^{1}$ and Alexander Maedche ${ }^{2}$ \\ ${ }^{1}$ INSEAD,CALT-Centre of Advanced Learning Technologies, 77300 Fontainebleau, France \\ liana.razmerita@ugal.ro, albert.angehrn@,insead.edu \\ ${ }^{2}$ FZI Research Center for Information Technologies, 76131 Karlsruhe, Germany \\ http://www.fzi.de/wim \\ maedche@,fzi.de
}

\begin{abstract}
This paper is presenting a generic ontology-based user modeling architecture, (OntobUM), applied in the context of a Knowledge Management System (KMS). Due to their powerful knowledge representation formalism and associated inference mechanisms, ontology-based systems are emerging as a natural choice for the next generation of KMSs operating in organizational, interorganizational as well as community contexts. User models, often addressed as user profiles, have been included in KMSs mainly as simple ways of capturing the user preferences and/or competencies. We extend this view by including other characteristics of the users relevant in the KM context and we explain the reason for doing this. The proposed user modeling system relies on a user ontology, using Semantic Web technologies, based on the IMS LIP specifications, and it is integrated in an ontology-based KMS called Ontologging. We are presenting a generic framework for implicit and explicit ontology-based user modeling.
\end{abstract}

\section{Introduction}

The knowledge-based theory of the firm suggests that knowledge is the organizational asset that enables sustainable competitive advantage in very dynamic and competitive markets. Knowledge is considered the most important asset for organizations and the effective management of knowledge has become an important issue. KMSs refer to a class of information systems applied to managing organizational knowledge [1]. Knowledge in the context of KMSs consists of experience, know-how and expertise of people (tacit knowledge) as well as different information artifacts, knowledge assets and data stored in documents, reports available within the organization and outside the organization (explicit knowledge).

Knowledge Management Systems are designed to allow users to access and utilize the rich sources of data, information and knowledge stored in different forms, but also to support knowledge creation, knowledge transfer and continuous learning for the knowledge workers. Recently KMSs, unlike databases, have aimed at going beyond the mere administration of electronic information; they now aim at fostering learning 
processes, knowledge sharing, collaboration between knowledge workers irrespective of their location, etc. KMSs tend to become complex in order to support the tasks mentioned above as they are not limited to providing easy access to knowledge assets and they address different categories of users with different needs, roles and preferences. Research on user modeling is motivated by two reasons: 1) differences in individual users' needs and 2) heterogeneity between different groups of users. Moreover user models and user modeling are the key element for personalized interaction and adaptive feature integration, two very important steps in developing advanced information systems.

The paper is organized as follows: section 2 provides an introduction of the overall ontology-based user modeling architecture. Subsequently, we provide details about explicit and implicit user modeling. In section 3 we discuss implementation details and in section 4 we conclude.

\section{Ontology-based User Modeling Architecture}

This section introduces the overall ontology-based user modeling (OntobUM) architecture. The overall user model for a specific user is based on an explicit definition provided by the user through the user profile editor (UPE) and by an implicit part maintained by intelligent services, as represented in Figure 1.

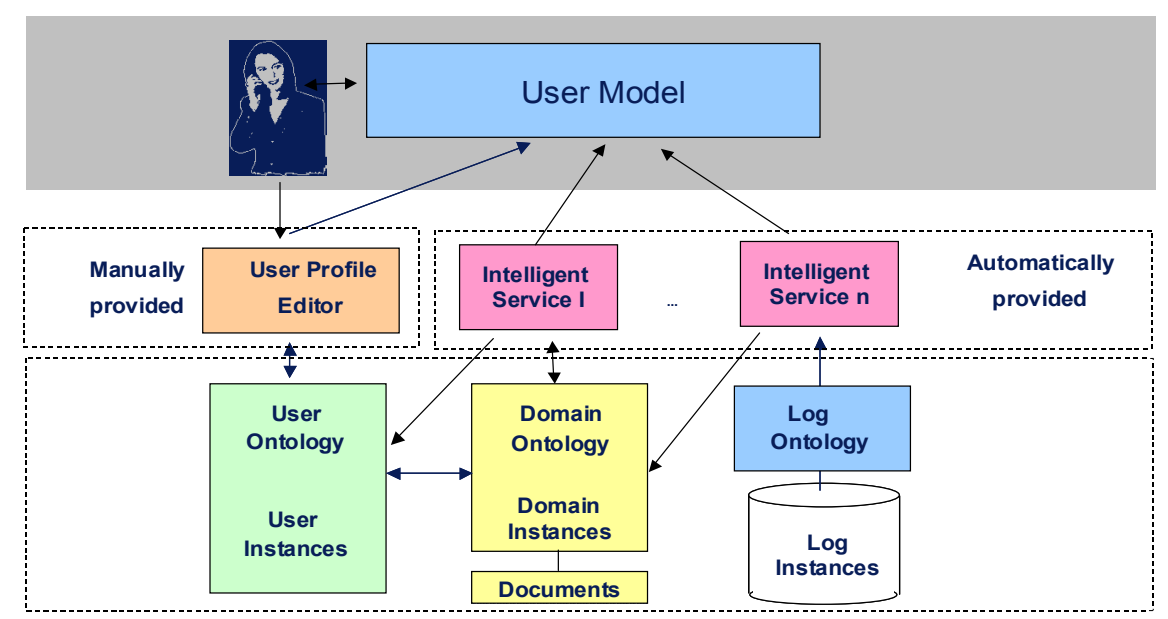

Figure 1 An ontology-based user modeling system

The intelligent services have two main roles in the system: (1) to update and maintain the user model on the basis of usage data through the application of a number of heuristics, (2) to provide personalized services based on the characteristics of the users (e.g. personalized views are generated and presented to the user based on the user's interests, background and role; notification agents announce new entries relevant to the user, etc). The system's general architecture enables one to add incrementally 
such intelligent services. The architecture of the ontology-based user modeling system integrates three different ontologies, as represented in Figure 1:

- The User Ontology structures the different characteristics of users and their relationships.

- The Domain Ontology defines the domain and application specific concepts and their relationships.

- The Log Ontology defines the semantics of the user interaction with the system. The log instances, or usage data, are generated by monitoring the user interaction with the system. Based on this usage data the system updates the user model and derives a user related behavior as described in section 2.2.

\subsection{The Explicit Part of the User Model}

The user profile editor UPE is a specialized ontology editor for user models. The UPE enables the user to enter user data but also to visualize them, revise them and update them afterwards. The definition of the user ontology captures rich metadata about the employee's profile including characteristics such as: identity, email, address, competencies, cognitive style, preferences, etc. but also a "behavioral profile" as described in the next section.

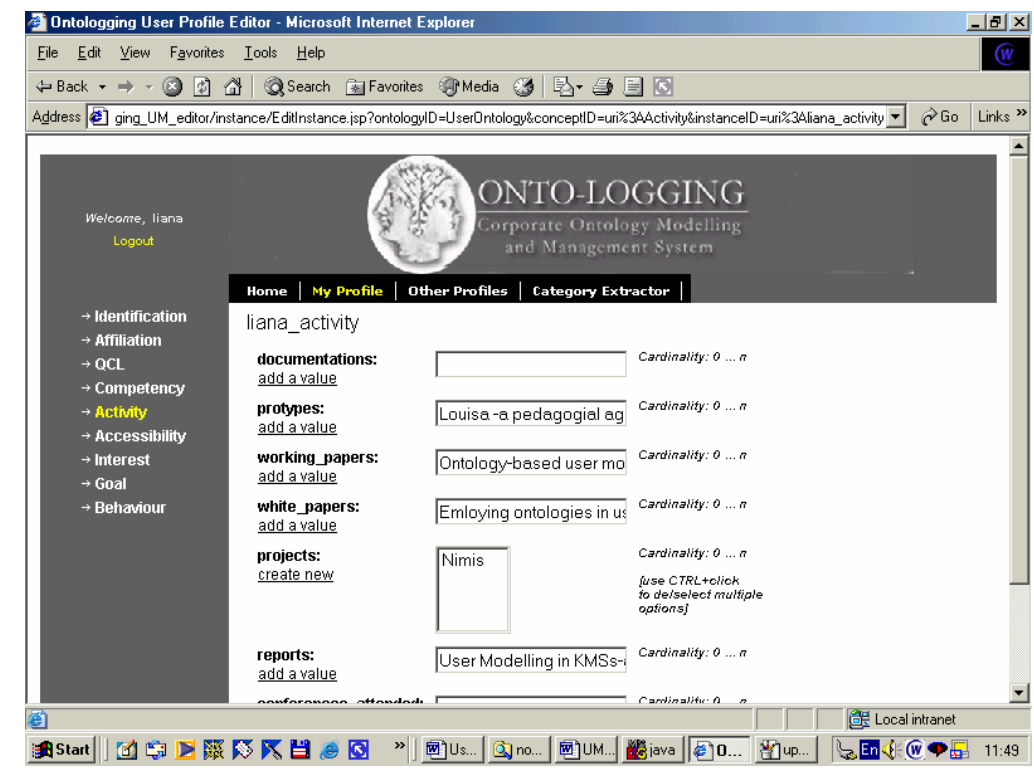

Figure 2 User profile editor

The proposed user model is structured according to Information Management Systems Learner Information Package specifications (IMS LIP). The IMS LIP package [2] is structured in eleven groupings including: Identification, Goal, QCL (Qualifications, Certification and Licenses), Accessibility, Activity, Competence, Interest, Af- 
filiation, Security Key and Relationship. Figure 2 shows a screenshot of the OntobUM's UPE, representing the Activity concept and its properties in the editing mode. UPE enables to switch from an edit mode to a view mode.

\subsection{The Implicit Part of the User Model}

KMSs need to encourage people to codify their experience, to share their knowledge and to develop an "active" attitude towards using the system. For this purpose we have extended the IMS LIP groupings with the Behavior concept. The Behavior concept and its subconcepts were introduced to "measure" two processes that are important for the effectiveness of a KMS, namely knowledge sharing and knowledge creation. The Behavior concept describes characteristics of users interacting with a KMS such as: level_of_activity, type_of_activity, level_of_knowledge_sharing, etc. Based on their activity in the system, namely the number of contributions to the system and the number of the documents read, OntobUM classifies the users into three stereotypes: readers, writers or lurkers. These categories are properties of the type_of_activity concept. The level_of_activity comprises four attributes that can be associated with the users: very active, active, passive or inactive. The classification of the users according to the type_of activity or level_of_activity is based on heuristics. For example a lurker is defined as somebody who doesn't contribute and who reads/accesses very few knowledge assets in the system. Several heuristics are dedicated to capture the interest areas and the level of expertise of the users. Through the level of knowledge sharing we are capturing the level of adoption of knowledge sharing practices. The user states in relation to the level of knowledge sharing are defined as: unaware, aware, interested, trial and adopter using Roger's terminology related to the attitude of people towards innovation (see [3]). Based on the identified characteristics, the system provides feedback, virtual reward or adapted interventions for a behavioural change (e.g. for adoption of knowledge sharing behaviour). In Razmerita et al. [4] we elaborate on how user models and user modeling can enhance the support in KMSs. We show how user models can be applied for: personalization, expertise discovery, networking, collaboration and learning.

\section{Implementation and related work}

In the area of user modeling, (Kay [5]; Chen and Mizoguchi, [6]) have already pointed out the advantage of using ontologies for learner/user models. Kay emphasizes the fact that a user model "needs an agreed ontology and representation so it can be used by different application programs".

OntobUM is a user-modeling server, which stores data in a RDF/RDFS format. Unlike other existing user modeling servers analyzed by Kobsa [7], OntobUM integrates Semantic Web technology. The system has been implemented as a web application using Java 2 (jdk 1.4). We have used the KAON - Karlsruhe Ontology and Semantic Web [8] framework as an API for managing ontologies. As a web ontology 
language, KAON extends RDF/RDFS, so the user ontology is RDF/RDFS compatible.

\section{Conclusions}

In this paper we address aspects of ontology-based user modeling and we present a generic architecture for modeling users based on ontologies. The main contribution of the paper consists in: (1) identifying aspects of user modeling relevant to KMSs, (2) integrating them in a generic framework based on ontologies. We give a concrete example of the use of the OntobUM framework in an ontology-based KMS, but this framework can be adapted to different ontology-aware environments. The user ontology is implemented using Semantic Web technology and it is structured on extended IMS LIP specifications. We have identified characteristics of the users that are relevant for a KMS under the Behaviour concept, but the largest part of the user ontology is generic and it could be reused in other application domains.

\section{Acknowledgment}

Research for this paper was partially financed by the EU in the IST-2000-28293 project Ontologging. We thank our Ontologging project partners for their contributions and their collaboration to this research work.

\section{References}

1. Leidner, D., Alavi, M., Review: knowledge management and knowledge management systems: conceptual foundations and research, INSEAD-MIS Quarterly, vol. 25 (no. 1), pp. 107-136, 2001.

2. IMS LIP, IMS Learner Information Package http://www.imsproject.org/aboutims.html, 2001

3. Angehrn, A., Nabeth, T., Leveraging Emerging Technologies in Management-Education: Research and Experiences, European Management Journal, Elsevier, 15, pp. 275-285, 1997.

4. Razmerita, L., Angehrn A., Nabeth, T., On the role of user models and user modeling in Knowledge Management Systems, in Proceedings of HCI International, Greece, to appear 2003.

5. Kay, J., Ontologies for reusable and scrutable student model, position paper, In Proceedings of AIED99 Workshop on Ontologies for Intelligent Educational Systems, 1999.

6. Chen, W. and Mizoguchi, R., Communication Content Ontology for Learner Model Agent in Multi-Agent Architecture, Proceedings of AIED99 Workshop on Ontologies for Intelligent Educational Systems, 1999.

7. Kobsa, A., Generic User Modeling Systems, in User Modeling and User-Adapted Interaction, 11(1-2), pp 49-63, 2001.

8. Maedche, A., Motik, B., Stojanovic, L., Studer, R. and Volz, R., Ontologies for Enterprise Knowledge Management, IEEE Intelligent Systems, November/December, 2002. 